\title{
An enhanced genetic model of relapsed IGH- translocated multiple myeloma evolutionary dynamics
}

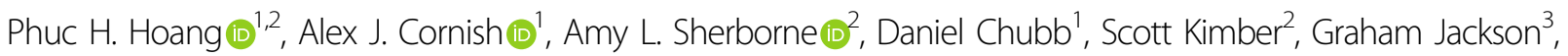

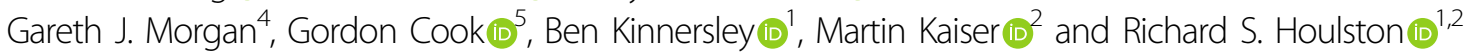

\begin{abstract}
Most patients with multiple myeloma (MM) die from progressive disease after relapse. To advance our understanding of MM evolution mechanisms, we performed whole-genome sequencing of $80 \mathrm{lGH}$-translocated tumour-normal newly diagnosed pairs and 24 matched relapsed tumours from the Myeloma XI trial. We identify multiple events as potentially important for survival and therapy-resistance at relapse including driver point mutations (e.g., TET2), translocations (MAP3K14), lengthened telomeres, and increased genomic instability (e.g., 17p deletions). Despite heterogeneous mutational processes contributing to relapsed mutations across MM subtypes, increased AID/APOBEC activity is particularly associated with shorter progression time to relapse, and contributes to higher mutational burden at relapse. In addition, we identify three enhanced major clonal evolution patterns of MM relapse, independent of treatment strategies and molecular karyotypes, questioning the viability of "evolutionary herding" approach in treating drug-resistant MM. Our data show that MM relapse is associated with acquisition of new mutations and clonal selection, and suggest APOBEC enzymes among potential targets for therapy-resistant MM.
\end{abstract}

\section{Introduction}

Multiple myeloma (MM) is caused by the expansion of clonal plasma cells in the bone marrow ${ }^{1}$. Over half of MM tumours have chromosomal translocations involving the immunoglobulin heavy chain locus, which leads to overexpression of oncogenes (CCND1, CCND3, MAF, MAFB, WHSC1/MMSET, and FGFR3) as an initiating event ${ }^{1}$. Despite recent advances, $M M$ is essentially an incurable malignancy, and most patients die from progressive disease after multiple relapses irrespective of treatment. Our limited knowledge of the molecular changes associated with relapse is a barrier to developing new therapeutic strategies to overcome drug resistance.

Correspondence: Martin Kaiser (Martin.Kaiser@icr.ac.uk) or

Richard S. Houlston (Richard.Houlston@icr.ac.uk)

'Division of Genetics and Epidemiology, The Institute of Cancer Research, London SM2 5NG, UK

${ }^{2}$ Division of Molecular Pathology, The Institute of Cancer Research, London SM2 5NG, UK

Full list of author information is available at the end of the article
To advance our understanding of the evolution of MM tumours and the mutational mechanisms that shape their history, we performed whole-genome sequencing (WGS) of 80 newly diagnosed MM tumour-normal pairs, 24 also had matched relapsed tumours ${ }^{2}$. WGS allowed us to examine the impact of noncoding mutations, complex structural rearrangements, and telomere structure on MM tumourigenesis analyses not possible in previous studies, which have been based on whole-exome sequencing (WES) ${ }^{3,4}$. Integrating information from multiple types of genomic alterations has allowed us to infer the order of mutational events, and show that relapse is associated with acquisition of new mutations and clonal selection.

\section{Materials and methods \\ Samples}

Bone marrow aspirates and blood samples were obtained from 80 patients with newly diagnosed MM

\section{(c) The Author(s) 2020}

(c) (i) Open Access This article is licensed under a Creative Commons Attribution 4.0 International License, which permits use, sharing, adaptation, distribution and reproduction cc) in any medium or format, as long as you give appropriate credit to the original author(s) and the source, provide a link to the Creative Commons license, and indicate if changes were made. The images or other third party material in this article are included in the article's Creative Commons license, unless indicated otherwise in a credit line to the material. If material is not included in the article's Creative Commons license and your intended use is not permitted by statutory regulation or exceeds the permitted use, you will need to obtain permission directly from the copyright holder. To view a copy of this license, visit http://creativecommons.org/licenses/by/4.0/. 
being treated according to the UK National Cancer Research Institute Myeloma XI trial protocol ${ }^{2}$. Matched relapsed tumour DNAs were available for 24/80 primary patients. Tumour DNAs were extracted from plasma cells selected and sorted using CD138 microbeads as described, previously ${ }^{5}$. In all cases tumour purity was in excess of $30 \%$. Germline DNA was derived from matched blood samples. Tumour $I G H$-translocation status was determined using multiplexed real-time $\mathrm{PCR}^{6}$. Hyperdiploid MM was defined as gain of at least two chromosomes as defined previously ${ }^{5}$. An entire chromosome was considered amplified if at least $90 \%$ of the chromosome overlapped with an amplification ${ }^{7}$. Clinical data and informed consent was obtained from all patients. Ethical approval for the study was obtained by the Oxfordshire Research Ethics Committee (MREC 17/09/09, ISRCTN49407852).

\section{Whole genome sequencing}

Sequencing libraries were prepared using Illumina SeqLab specific TruSeq Nano High Throughput library preparation kit (Illumina Inc, San Diego, CA 92122 USA), and paired end sequencing was conducted using Illumina HiSeqX technology. Raw WGS sequencing data were quality checked using FastQC (v.0.11.4) and aligned using the Burrows-Wheeler Alignment tool ${ }^{8}$ (BWA v0.7.13) to the human genome hg38 assembly using default parameters. Matching of tumour, normal, and relapsed samples was confirmed using NGSCheckMate ${ }^{9}$. Single nucleotide variants (SNVs) and indels were called using MuTect2 (v4.0.3.0) ${ }^{10}$ according to best practices, using The Genome Aggregation Database (gnomAD) ${ }^{11}$ file in GRCh38 provided as part of the GATK resource. Variants were filtered for cross-sample contamination, oxidation artefacts $^{10}$, quality score ${ }^{7}$, and using a panel of normals generated from 80 germline samples. Variants with a germline population allele frequency $>0.1 \%$ in gnomAD or in repetitive regions defined by University California Santa Cruz (UCSC) were excluded. Somatic indels were excluded if they were supported by $<20 \%$ of tumour sample reads overlapping the position ${ }^{12}$ or were located within ten base pairs of a germline indel catalogued by gnomAD.

Reconstruction of clonal and subclonal copy number alterations (CNAs) for primary and relapsed tumours was conducted using Battenberg ${ }^{13}$. Since copy-neutral loss of heterozygosity $(\mathrm{nLOH})$ is intrinsically more problematic to identify accurately ${ }^{14}$, these segments called by Battenberg were inspected manually against CNA calls overlapping within $10 \mathrm{Mb}$ of two other CNA callers Sequenza ${ }^{15}$ and FACETS ${ }^{16}$. The copy number status of an $\mathrm{nLOH}$ segment was corrected and only reported if it was supported by at least two of the three CNA callers, and was excluded from downstream analysis if all methods were discordant. Tumour purity estimated by Battenberg was compared against and corrected using Ccube ${ }^{17}$. Somatic structural variants (SVs) were identified taking a consensus approach, as implemented by The Pancancer Analysis of Whole Genomes ${ }^{18}$, considering only variants identified by at least two of MANTA (v1.2.0) ${ }^{19}$, LUMPY $(\mathrm{v} 0.2 .13)^{20}$, or DELLY (v0.7.9) ${ }^{21}$. Chromothripsis regions were identified using ShatterSeek, adopting the criteria of at least four adjacent segments oscillating copy number states and at least six interleaved $\mathrm{SVs}^{22}$. All candidate chromothripsis regions were manually curated as previously advocated ${ }^{22}$. Chromoplexy was detected using ChainFinder (v1.0.1) with default parameters ${ }^{23}$ and hg38 UCSC cytoband definitions (http://hgdownload.cse.ucsc. edu/goldenpath/hg38/database/). As previously advocated $^{22}$, chromoplexy was only called when at least three chromosomes were involved in a chain of SVs. Telomere length was estimated using Telomerecat ${ }^{24}$ with default parameters. Kataegis foci were identified using the KataegisPortal with default parameters (https://github. com/MeichunCai/KataegisPortal), and defined as having six or more consecutive mutations with an average mutational distance $\leq 1 \mathrm{~Kb}$, excluding immune hypermutated regions ${ }^{25}$.

\section{Identifying driver mutations}

Coding drivers were identified using $\mathrm{dNdScv}$ with default parameters ${ }^{26}$. Nonsilent mutations in a curated list of 82 established coding drivers ${ }^{7,27}$ and all coding genes were compared in matched primary and relapsed tumours. To identify noncoding drivers we analysed promoter and cis-regulatory regions (CREs) as described previously ${ }^{7}$. Briefly, promoters were defined as intervals spanning $400 \mathrm{bp}$ upstream and $250 \mathrm{bp}$ downstream of transcription start site from GENCODE (release 25$)^{28}$. CREs were defined using promoter capture $\mathrm{Hi}-\mathrm{C}$ data generated on naïve $\mathrm{B}$-cells ${ }^{29}$. Raw sequencing reads from European Genome-Phenome Archive (EGA; accession code EGAS00001001911) were aligned to hg38 using HiCUP (v0.6.1) ${ }^{30}$ and promoter-CRE interactions were called with CHiCAGO (v1.8) ${ }^{31}$. Only interactions with linear distance $\leq 1 \mathrm{Mb}$ and $\mathrm{CHiCAGO}$ score $\geq 5$ were considered ${ }^{7}$.

Recurrently mutated promoters and CREs were identified using a Poisson binomial model as previously described $^{7,32}$, taking into account tumour ID, trinucleotide context, and replication timing. For CRE regions, mutations were excluded if they overlap with open reading frames, $5^{\prime}$-UTR, and $3^{\prime}$-UTR as defined by Ensembl ${ }^{7}$. For promoters, mutations overlapping with open reading frames were excluded. Replication timing was estimated as the average of two B-lymphocyte replicates ${ }^{33,34}$. For promoters and CREs mutated in $\geq 3$ samples, the clustering of mutations was examined using a permutation 
approach considering the number of mutations occurring at the same nucleotide position as previously described ${ }^{7}$. For each promoter and CRE, a combined $P$-value from the mutational recurrence and clustering analyses were obtained using Fisher's method $^{7,35}$. The Benjamini-Hochberg false discovery rate (FDR) procedure was used to adjust for multiple testing with significant threshold at $Q<0.05$. Promoters and CREs overlap with immune hypermutated regions were excluded to avoid false positives. We only report CREs and promoters mutated in at least three tumours.

\section{Impact of cereblon and IMiD response pathway genes mutation on relapse}

All patients we studied were treated with immunomodulatory drugs (IMiDs), either thalidomide or lenalidomide. Mutations in CRBN and associated genes have been proposed as being a mechanism of acquired drug resistance to IMiDs ${ }^{36,37}$. To examine this proposition, we specifically considered nonsynonymous mutations, CNAs, and SVs disrupting a curated list of $42 \mathrm{CRBN} / \mathrm{IMiD}$ genes-genes involved in the CRBN pathway regulation and IMiD response (Supplementary Table 1).

\section{Chronology of mutational events}

The chronological timing of SNVs and CNAs was estimated independently for the 80 primary tumours as previously described ${ }^{38}$. Briefly, for SNVs we considered only driver genes mutated in $\geq 4$ samples to allow reliable estimation of relative timing. For CNAs we considered only large-scale autosomal events $(\geq 3 \mathrm{Mb})$ present in $\geq 8$ samples $^{38}$. Cytobands were assigned based on UCSC hg38 definitions. One sample (8573) displayed hyperdiploid characteristics and was excluded from the analysis. Cancer cell fractions (CCFs) of each CNV event and SNV were estimated using Battenberg ${ }^{13}$. Each cytoband or driver gene was ordered by mean of CCF from highest to lowest. The Tukey's range test and a stepwise approach were used to test for difference between the CCF means of consecutive cytobands or driver genes to define discrete clonality levels, as described previously ${ }^{38}$. As previously advocated $^{38}, 95 \%$ confidence intervals were calculated with basic bootstrap method with 1000 iterations using boot $\mathrm{R}$ package.

\section{Analysis of copy number changes}

Permutation was used to test the null hypothesis that the frequency of particular chromosome arm copy number events does not differ between primary and relapse MM. We first counted change in frequency of affected tumours at primary and relapse. We then randomly swapped condition labels for all matched primary and relapsed tumours 10,000 times, and recounted change in chromosome arm event frequency. Empirical $P$-values for each chromosome arm event were calculated as fraction of permutations with absolute net frequency change at least as great as the absolute net frequency change observed in the true primary/relapse labelling. We only considered chromosome arm events with net change in frequency in at least two tumours.

We employed a permutation-based approach to test the null hypothesis that additional relapse-associated CNA events occur by chance at pre-existing unstable genomic regions. For each autosomal chromosome arm, we counted the number of tumours with additional largescale CNA on the considered chromosome arm at relapse. The tested chromosome arm in considered tumours with further CNA change were permutated 10,000 times among 44 possible chromosome arms loci (22 autosomal chromosomes with either $\mathrm{p}$ or $\mathrm{q}$ arm). The empirical $P$ values were calculated as the fraction of permutations with the number of additional CNA change were at least as great as the original tested chromosome arm.

\section{Mapping evolutionary trajectories}

Analysis of clonality was conducted using only SNVs in diploid regions, as miscalled copy number states can confound the analysis. Potential neutral tail mutations were identified using MOBSTER ${ }^{39}$ and excluded prior to clustering procedure to minimise calling false positive clones. For each primary and relapse tumour pair, we performed two-dimensional variant clustering using a Bayesian Dirichlet process implemented in DPclust ${ }^{3,13}$. Only those clusters with $\geq 1 \%$ of total mutations and $\geq 100$ SNVs were considered. Muller plots were generated with Timescape $\mathrm{R}$ package version 1.10.0. For each cluster in primary tumour and matched relapse, the proportion of SNVs shared was calculated.

\section{Mutational signatures}

De novo extraction of signatures was performed on 80 primary and 24 relapsed genomes separately using nonnegative matrix factorization ${ }^{40}$. We compared de novo mutational signatures with Catalogue of Somatic Mutations in Cancer (COSMIC) single base substitution (SBS) signatures version 3 by computing their cosine similarities $^{41}$. A de novo mutational signature was assigned to a COSMIC signature if the cosine similarity was $>0.75$ as advocated $^{12}$. We next performed signature fitting using deconstructSigs ${ }^{42}$ considering only those COSMIC signatures extracted de novo, as previously recommended ${ }^{43}$. In view of potential ambiguous assignment, we combined the contributions of the flat profile signatures 5,8 , and $40^{25,42,43}$, excluding signature 3 as this signature is unlikely to be active in $\mathrm{MM}^{43}$. As previously advocated, we compared mutational signature proportions in paired primary and relapsed samples using the chi-squared test $^{13}$. Association between changes in mutational 
burden and AID/APOBEC mutational contribution for paired primary and relapsed tumours was calculated using Fisher's exact test. Spearman correlation was performed to test the association between AID/APOBEC contribution of relapse-specific mutations and time to relapse.

\section{Results}

We carried out WGS on 80 newly diagnosed MM tumour-normal pairs from the Myeloma XI trial, and matched relapsed tumour from 24 patients. The 80 patients had either $t(4 ; 14)(n=38), t(11 ; 14)(n=38)$, or $t$ $(14 ; 16)(n=4) \mathrm{MM}$, with one patient carrying both $t(4 ; 14)$ translocation and trisomy of chromosomes 9 and 15 (Table 1). Hyperdiploid (HD) and non-HD subtypes of MM have distinctive genomic landscapes and are a priori likely to have different evolutionary trajectories ${ }^{1}$. In this study, we restricted our analysis to $I G H$-translocated tumours to focus on examining evolutionary dynamics of non-HD myeloma. WGS resulted in a median of $38 \times$ coverage for normal samples $(30-44 \times), 111 \times$ for primary tumours $(82-155 \times)$, and $114 \times$ for the 24 relapsed tumours (102-154x) (Supplementary Table 2). 6 of the 80 patients have been the subject of a previous WES project ${ }^{4}$.

\section{Mutational events in primary tumours}

We began by surveying for important genetic alterations in the 80 primary MM tumours by considering the contribution of both protein-coding and noncoding SNVs and indels, as well as CNAs. As expected, significantly mutated genes $(Q<0.05)$ at presentation were DIS3, KRAS, NRAS, FGFR3, MAX, CCND1, TP53, IRF4, and PRKD2 (Fig. 1a and Supplementary Table 3). The promoters of 17 genes including BCL6, CXCR4, BIRC3, MYO1E, CRIP1, FLT3LG, and DPP9 were also significantly mutated as well as nine cis-regulatory elements (CREs) interacting with genes including PAX5, BCL6, ZCCHC7, and IFNGR1 (Supplementary Fig. 1 and Supplementary Tables 4,5$)$. The most frequent large-scale CNAs were deletion of 13q (73\%), 22q (35\%), and $1 p$ (35\%); and gain of 1q (45\%). (Fig. 1a and Supplementary Fig. 2 and Supplementary Table 6). Aberrations of 13q was enriched in high-risk $t(4 ; 14)$ and $t(14 ; 16) \mathrm{MM}(P=$ $3.5 \times 10^{-5}$, odd ratio $=16.2$, Fisher's exact test).

Chromothripsis was observed in $18 / 80$ primary tumours (23\%) with the most frequently affected chromosomes are 1 (4 tumours), 8, 11, and 22 (3 tumours) (Supplementary Fig. 3); whereas $3 \%(2 / 80)$ of primary tumours featured chromoplexy (Supplementary Fig. 4). The frequency of chromothripsis and chromoplexy identified is comparable to a previous report ${ }^{44}$. Chromoplexy resulted in the simultaneous disruption of multiple driver genes ${ }^{7,27}$ (KRAS, PRKD2, PTPN11, PTH2, BAX, CELA1, FTL, $A R I D 2$, and $C D K N 1 B$ ) in primary tumours. Overall across the 80 primary tumours, high-risk subtypes MM $t(4 ; 14)$ and $\mathrm{t}(14 ; 16)$ were associated with a shorter telomeres $\left(P=9.2 \times 10^{-5}\right.$, Wilcoxon rank-sum test) (Supplementary Fig. 5).

By integrating somatic mutations and copy number profiles we inferred the relative timing of key driver alterations in $\mathrm{MM}$ (i.e., which events occur earlier relative to others). Mutations of CCND1, MAX, PRKD2, DIS3, and $N R A S$ were identified as early events whereas mutations of KRAS, IRF4, FGFR3, TP53, and TET2 occurred as later events (Fig. 1b). Chronological timing of major CNAs (present in $\geq 10 \%$ of total samples) $^{38}$ identified $21 \mathrm{q}$ gain and 13q deletion as being early events (Fig. 1c), consistent with a previous report that $13 q$ deletions tend to be clo$\mathrm{nal}^{45} .1 \mathrm{p}$ deletion and $1 \mathrm{q}$ gain, which has been linked to patient prognosis were identified as later events (Fig. 1c).

\section{Mutational landscape of relapse}

We next investigated the molecular features of $\mathrm{MM}$ relapse by analysis of the 24 primary-relapse pairs. Patients received cyclophosphamide and dexamethasone in combination with either thalidomide (CTD), lenalidomide (RCD), or both carfilzomib and lenalidomide (CCRD) as induction therapy. Fit and young patients received high-dose melphalan (intensive pathway). 9 of the 25 patients subsequently received lenalidomide maintenance therapy. Treatment histories of each patient are summarized in Table 1. None of the patients we studied had detectable $C R B N$ mutations at relapse. We did, however observe increased IKZF3 mutation CCF and de novo mutations disrupting CRBN/IMiD genes in two patients at relapse$R B X 1$ mutation and copy number loss affecting $U B E 2 A$ (Supplementary Table 7). Relapse was associated with a higher mutational burden than primary tumours (Supplementary Fig. 6a-b, $P<0.01$, paired Wilcoxon rank-sum test). Varied proportions (9-63\%) of SNVs and indels identified in primary tumours were not detectable at relapse (Supplementary Fig. 6c), suggesting eradication and heterogenous clonal dynamics of the respective clone. Despite the increased mutational burden, relapsed tumours did not exhibit significantly more kataegis (Supplementary Fig. 7 and Supplementary Table 8). Chromothripis and chromoplexy were each observed in only one additional relapsed tumour (7842 and 8237 respectively; Supplementary Figs. 8 and 9). Although both primary and relapsed tumours had shorter telomeres compared to plasma cells $(P<0.01$, paired Wilcoxon rank-sum test), relapse was associated with longer telomeres $\left(P=5.3 \times 10^{-3}\right)$ (Supplementary Fig. 10).

A translocation bringing the $I G H$ loci in proximity to MAP3K14 was gained at relapse in one tumour (Supplementary Fig. 11). Driver genes additionally mutated at relapse included $F A M 46 C$, TRAF2, LTB, FAM154B, NF1, $X B P 1$, and $I D H 2$ (Supplementary Fig. 12). Driver mutations most frequently acquired at relapse were those in KRAS and NRAS, detected in three and two tumours 
Table 1 Summary of demographic and treatment data.

\begin{tabular}{|c|c|c|c|c|c|c|c|}
\hline Sample ID & Karyotype & Gender & Age & Elapsed time (months) & Induction & Maintenance & Pathway \\
\hline 1305 & $11 ; 14$ & Male & 51 & 38.34 & CTD & No & Intensive \\
\hline 1334 & $11 ; 14$ & Female & 43 & 24.00 & CTD & Missing & Intensive \\
\hline 5834 & $11 ; 14$ & Female & 69 & 29.93 & CTDa & No & Nonintensive \\
\hline 6030 & $4 ; 14$ & Female & 36 & 19.75 & CTD & No & Intensive \\
\hline 6178 & $11 ; 14$ & Female & 67 & 18.40 & $\mathrm{RCD}$ & Missing & Intensive \\
\hline 6229 & $11 ; 14$ & Male & 74 & 9.23 & CTDa & Missing & Nonintensive \\
\hline 6706 & $11 ; 14$ & Male & 59 & 25.43 & $\mathrm{RCD}$ & No & Intensive \\
\hline 6988 & $11 ; 14$ & Male & 69 & 12.26 & $\mathrm{RCDa}$ & No & Nonintensive \\
\hline 7020 & $4 ; 14$ & Female & 58 & 14.69 & CTD & Missing & Intensive \\
\hline 7240 & $4 ; 14$ & Male & 55 & 11.30 & RCD & Lenalidomide & Intensive \\
\hline 7801 & $14 ; 16$ & Female & 48 & 14.49 & CTD & Missing & Intensive \\
\hline 7842 & $4 ; 14$ & Male & 66 & 17.64 & CTD & No & Intensive \\
\hline 8237 & $4 ; 14$ & Female & 49 & 14.00 & CTD & No & Intensive \\
\hline 9126 & $11 ; 14$ & Male & 64 & 16.23 & CTDa & Missing & Nonintensive \\
\hline 9166 & $14 ; 16$ & Female & 68 & 27.24 & CCRD & No & Intensive \\
\hline 9515 & $11 ; 14$ & Male & 68 & 26.15 & $\mathrm{RCDa}$ & Lenalidomide & Nonintensive \\
\hline 9721 & $14 ; 16$ & Male & 64 & 29.44 & CTD & Lenalidomide & Intensive \\
\hline 10,068 & $4 ; 14$ & Male & 71 & 13.77 & RCDa & Lenalidomide and Vorinostat & Nonintensive \\
\hline 10,365 & $11 ; 14$ & Male & 76 & 9.33 & CTD & Missing & Intensive \\
\hline 11,506 & $14 ; 16$ & Male & 77 & 11.83 & CTDa & Lenalidomide & Nonintensive \\
\hline 11,668 & $4 ; 14$ & Male & 49 & 19.29 & $\mathrm{RCDa}$ & Missing & Nonintensive \\
\hline 11,949 & $11 ; 14$ & Male & 76 & 14.65 & CTD & Missing & Intensive \\
\hline 12,546 & $4 ; 14$ & Male & 77 & 30.59 & RCD & Missing & Intensive \\
\hline 13,029 & $4 ; 14$ & Male & 62 & 6.90 & CTD & Missing & Intensive \\
\hline 5695 & $11 ; 14$ & Male & 64 & NA & CTD & No & Intensive \\
\hline 5699 & $11 ; 14$ & Female & 68 & NA & CTD & Missing & Intensive \\
\hline 5836 & $11 ; 14$ & Male & 77 & NA & CTDa & No & Nonintensive \\
\hline 5939 & $4 ; 14$ & Male & 65 & NA & CTD & Missing & Intensive \\
\hline 6016 & $11 ; 14$ & Female & 55 & NA & $\mathrm{RCD}$ & Missing & Intensive \\
\hline 6076 & $4 ; 14$ & Male & 72 & NA & RCDa & Lenalidomide & Nonintensive \\
\hline 6163 & $4 ; 14$ & Male & 75 & NA & $\mathrm{RCDa}$ & Missing & Nonintensive \\
\hline 6277 & $11 ; 14$ & Male & 56 & NA & $\mathrm{RCD}$ & Lenalidomide & Intensive \\
\hline 6279 & $4 ; 14$ & Male & 62 & NA & $\mathrm{RCD}$ & Lenalidomide & Intensive \\
\hline 6345 & $4 ; 14$ & Female & 72 & NA & CTDa & Missing & Nonintensive \\
\hline 6415 & $11 ; 14$ & Female & 68 & NA & $\mathrm{RCDa}$ & Missing & Nonintensive \\
\hline 6425 & $4 ; 14$ & Male & 67 & NA & RCD & Lenalidomide and Vorinostat & Intensive \\
\hline 6501 & $11 ; 14$ & Female & 51 & NA & $\mathrm{RCD}$ & Missing & Intensive \\
\hline 6702 & $4 ; 14$ & Female & 78 & NA & CTDa & Missing & Nonintensive \\
\hline 7000 & $11 ; 14$ & Female & 78 & NA & CTDa & Missing & Nonintensive \\
\hline 7005 & $4 ; 14$ & Male & 74 & NA & CTDa & Missing & Nonintensive \\
\hline 7164 & $11 ; 14$ & Female & 80 & NA & $\mathrm{RCDa}$ & Missing & Nonintensive \\
\hline 7348 & $4 ; 14$ & Male & 67 & NA & $\mathrm{RCDa}$ & No & Nonintensive \\
\hline 7729 & $4 ; 14$ & Male & 65 & NA & RCD & Lenalidomide and Vorinostat & Intensive \\
\hline 7794 & $4 ; 14$ & Female & 52 & NA & CTD & No & Intensive \\
\hline 7880 & $4 ; 14$ & Female & 82 & NA & $\mathrm{RCDa}$ & Missing & Non-intensive \\
\hline 7915 & $4 ; 14$ & Male & 59 & NA & CTD & Lenalidomide and Vorinostat & Intensive \\
\hline 7925 & $4 ; 14$ & Male & 59 & NA & CTD & Missing & Intensive \\
\hline 7950 & $4 ; 14$ & Male & 49 & NA & CTD & Lenalidomide and Vorinostat & Intensive \\
\hline 7956 & $4 ; 14$ & Female & 56 & NA & CTD & Missing & Intensive \\
\hline 8043 & $4 ; 14$ & Female & 81 & NA & CTDa & Missing & Non-intensive \\
\hline 8245 & $11 ; 14$ & Female & 63 & NA & $\mathrm{RCD}$ & Lenalidomide & Intensive \\
\hline 8567 & $11 ; 14$ & Female & 66 & NA & $\mathrm{RCDa}$ & Lenalidomide and Vorinostat & Nonintensive \\
\hline 8573 & $4 ; 14 / H D$ & Female & 82 & NA & CTDa & Missing & Nonintensive \\
\hline 8928 & $4 ; 14$ & Male & 52 & NA & CTD & Missing & Intensive \\
\hline
\end{tabular}


Table 1 continued

\begin{tabular}{|c|c|c|c|c|c|c|c|}
\hline Sample ID & Karyotype & Gender & Age & Elapsed time (months) & Induction & Maintenance & Pathway \\
\hline 8979 & $4 ; 14$ & Male & 76 & NA & CTDa & Missing & Nonintensive \\
\hline 9069 & $11 ; 14$ & Male & 73 & NA & $\mathrm{RCDa}$ & Missing & Non-intensive \\
\hline 9176 & $11 ; 14$ & Male & 78 & NA & $\mathrm{RCDa}$ & Missing & Nonintensive \\
\hline 9210 & $11 ; 14$ & Male & 69 & NA & CTD & Missing & Intensive \\
\hline 9249 & $11 ; 14$ & Male & 58 & NA & RCD & Lenalidomide & Intensive \\
\hline 9289 & $11 ; 14$ & Male & 56 & NA & CTD & No & Intensive \\
\hline 9292 & $4 ; 14$ & Female & 74 & NA & CTDa & Missing & Nonintensive \\
\hline 9337 & $11 ; 14$ & Female & 71 & NA & CTDa & Missing & Nonintensive \\
\hline 9376 & $4 ; 14$ & Female & 64 & NA & $\mathrm{RCD}$ & Missing & Intensive \\
\hline 9409 & $11 ; 14$ & Male & 73 & NA & CTDa & Missing & Nonintensive \\
\hline 9524 & $4 ; 14$ & Male & 51 & NA & $\mathrm{RCDa}$ & Lenalidomide & Nonintensive \\
\hline 9544 & $11 ; 14$ & Male & 67 & NA & $\mathrm{RCDa}$ & No & Nonintensive \\
\hline 9623 & $11 ; 14$ & Male & 58 & NA & $\mathrm{RCD}$ & Lenalidomide & Intensive \\
\hline 9718 & $4 ; 14$ & Male & 66 & NA & $\mathrm{RCDa}$ & No & Nonintensive \\
\hline 9917 & $11 ; 14$ & Male & 76 & NA & CTDa & Missing & Nonintensive \\
\hline 9931 & $11 ; 14$ & Female & 55 & NA & $\mathrm{RCD}$ & Missing & Intensive \\
\hline 10,085 & $11 ; 14$ & Female & 59 & NA & CCRD & Lenalidomide & Intensive \\
\hline 10,212 & $11 ; 14$ & Female & 79 & NA & $\mathrm{RCDa}$ & Lenalidomide & Nonintensive \\
\hline 10,597 & $4 ; 14$ & Male & 59 & NA & CCRD & No & Intensive \\
\hline 10,772 & $4 ; 14$ & Female & 63 & NA & CCRD & Missing & Intensive \\
\hline 10,801 & $11 ; 14$ & Male & 77 & NA & $\mathrm{RCDa}$ & Missing & Nonintensive \\
\hline 11,029 & $4 ; 14$ & Female & 73 & NA & $\mathrm{RCDa}$ & Missing & Nonintensive \\
\hline 11,897 & $4 ; 14$ & Male & 58 & NA & CCRD & Lenalidomide & Intensive \\
\hline 12,101 & $4 ; 14$ & Male & 62 & NA & CCRD & Missing & Intensive \\
\hline 12,227 & $11 ; 14$ & Male & 57 & NA & CCRD & No & Intensive \\
\hline 12,541 & $11 ; 14$ & Male & 56 & NA & CTD & Missing & Intensive \\
\hline
\end{tabular}

CTD cyclophosphamide, thalidomie, and dexamethasone, CTDa CTD with a reduced dose of dexamethasone and lower starting dose of thalidomide, $R C D$ Lenalidomide (Revlimid), cyclophosphamide, and dexamethasone, RCDa RCD with a reduced dose of dexamethasone, CCRD carfilzomib, Cyclophosphamide, lenalidomide, and dexamethasone. Intensive pathway: treatment with high dose melphalan after induction. NA: Matched relapsed data are not available.

respectively. The increase in CCF of TET2 mutations implied selection of subclones (Supplementary Fig. 13). The promoters and CREs of an additional 16 genes were significantly mutated at relapse, including genes with established roles in the biology of $\mathrm{MM}$ or other B-cell malignancies such as XBP1, BCL7A, and BCL9 (Supplementary Tables 9 and 10).

Relapse was associated with additional CNAs, most frequently for $17 \mathrm{p}$ deletion $\left(P<2.2 \times 10^{-6}\right)$ (Fig. 2a, Supplementary Fig. 14, and Supplementary Table 11). We observed additional CNAs occurring at pre-existing unstable genomic regions, including the progression of copy-neutral loss of heterozygosity (nLOH) to LOH, LOH to complete deletion; as well as further copy number gains (Fig. 2b and Supplementary Fig. 15). Such trend was observed at a higher rate than expected by chance at $11 \mathrm{q}$ $(P=0.042)$ and $14 \mathrm{q}(P=0.023)$ (Fig. 2c).

\section{Mutational processes active at relapse}

At diagnosis, the major mutational signatures in tumours were those indicative of aging (SBS5), AID/ APOBEC (SBS2, 9, and 13), and flat signatures (SBS5, 8, and 40) as previously observed ${ }^{7,25}$ (Supplementary Figs. 16 and 17). No additional mutational signatures potentially specific to treatment were extracted at relapse (Supplementary Fig. 18). Across all patients, we observed heterogeneous dynamic of mutational processes contributing to relapse (Supplementary Fig. 19). However, tumours with increased mutational burden at relapse were often associated with increased AID/APOBEC enzymes activity $(P=0.061$, Fisher's exact test). Despite the enrichment of APOBEC signatures in $t(14 ; 16) \mathrm{MM}$ $(P=0.017$, Wilcoxon rank-sum test) (Supplementary Fig. 17), we did not observe specific association of the signatures at relapse in this subtype $(P=0.20$, Wilcoxon rank-sum test), consistent with previous finding ${ }^{46}$. Notably, patients with higher AID/APOBEC mutational contribution at relapse were associated with shorter refractory time $(r=-0.43, P=0.037$, Spearman correlation) (Supplementary Figure 20). An increased $C \cdot G>G \cdot C$ transversion rate in relapse-specific mutations was also observed $(Q=0.015$, paired Wilcoxon rank-sum tests) (Supplementary Fig. 21), a feature previously reported in relapsed acute myeloid leukaemia ${ }^{47}$. 


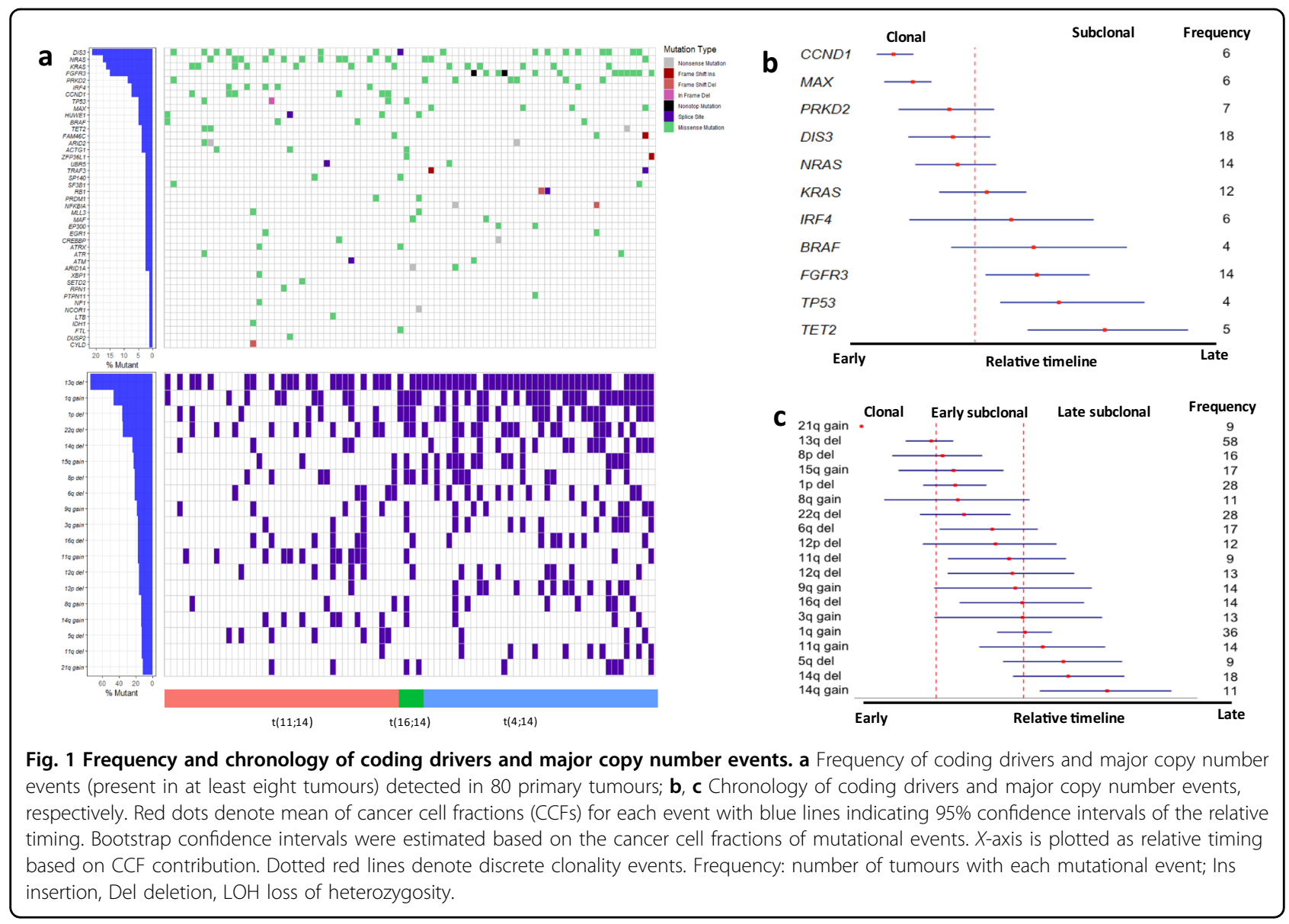

\section{Evolutionary trajectories of relapse}

Three patterns of clonal evolution were apparent at relapse (Fig. 3). In Pattern 1 (3/24 patients), the dominant clone in primary survives treatment and gains additional mutations at relapse (Fig. 3a, Supplementary Fig. 22a). Tumours with Pattern 1 are characterised with no change in clonal composition of the dominant clones, suggesting that they were potentially unaffected by treatment. Pattern $2(4 / 24$ patients) is featured by subclonal expansion whereby a subclone in the primary survives treatment, and expands to become the dominant clone at relapse (Fig. 3b and Supplementary Fig. 22b). Tumours with Pattern 2 are also accompanied with "branching evolution" feature, where new clones emerge while others are lost. We suspect these clones might have mutations (e.g., TET2 and $6 \mathrm{q}$ deletion) giving them survival and selective advantage. Pattern 3 (17/24 patients) is characterised by the emergence of new clones at relapse, accompanied by the disappearance or decline of primary clones (Fig. 3c and Supplementary Fig. 22c). The three patterns of clonal evolution were not associated with therapy strategies (intensive versus nonintensive pathways) or molecular karyotypes (Fisher's exact test). It was, however, of note that time to relapse was shorter with Pattern 2 (median 11.6 versus 19.3 months, $P=0.019$, Wilcoxon rank-sum test).

\section{Discussion}

Using high-depth WGS, we provide for an enhanced genetic model of the development and progression of MM. Our study expands upon previous findings, which have been based on WES/targeted sequencing $3,4,36,46,48,49$, low coverage WGS ${ }^{50}$, or fluorescence in situ hybridization and/or array technology ${ }^{46,51}$. While we have restricted our analysis to $\mathrm{MM}$ with an initiating translocation, our findings provide evidence for a common origin of tumour subpopulations with many tumours being composed of at least one subclone, reflecting the clonal heterogeneity present in both primary and relapse.

In addition to known coding drivers, we extend the number of potential non-coding drivers in $\mathrm{MM}$, including those associated with CXCR4 and BIRC3. Somatic mutations in $B C L 6$ promoters are common in $\mathrm{MM}^{52}$; however, since the gene is a common target of normal activationinduced deaminase (AID) in the germinal centre ${ }^{53}$, the relevance of these promoter mutations to MM biology is questionable. Noncoding regulatory regions additionally disrupted at relapse, included those targeting $X B P 1$, 


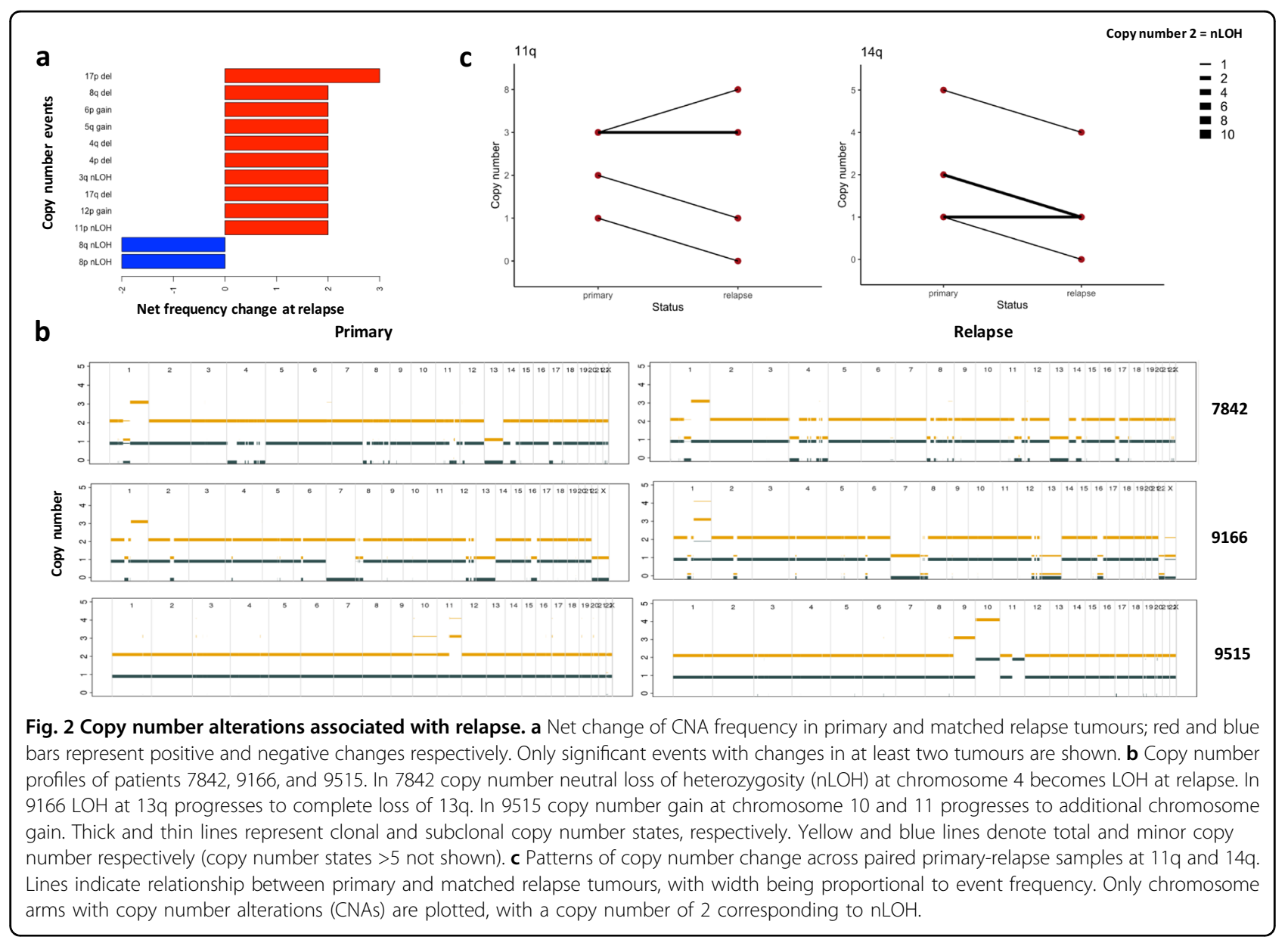

$R B X 1$, and $S C M L 1$. Common pathways affected by coding and noncoding mutations arising in MM relapse included those associated with WNT-signalling, MAPK-signalling, and NOTCH-signalling, base excision repair, cell cycle, telomere maintenance, and cellular senescence (Table 2). Notably, relapse was characterised by frequent additional CNAs, the most common being $17 p$ deletion. Since the additional CNAs often occurred at unstable genomic regions such as $11 \mathrm{q}$ and $14 \mathrm{q}$, it suggests increased chromosome instability are important means to escape therapy, analogous to that seen with chronic myeloid leukaemia in response to imatinib ${ }^{54}$. Our findings suggest that $21 \mathrm{q}$ gain, $13 \mathrm{q}$ deletion, and mutation of CCND1, $M A X, P R K D 2, D I S 3$, and NRAS are early events. The chronology of coding events identified from our study are broadly consistent with previous WES-based analyses ${ }^{1,55,56}$, any discrepancies are likely to be a consequence of sample size, representation of MM subtype, and number of coding drivers considered.

Overall, the mutational load was higher in relapse MM and aberrations previously linked to MM resurfaced in both primary pretreatment and relapse tumours in our cohort, including mutations in RAS genes, DIS3, TP53,
FGFR3, and PAX5 CRE mutations. As well as highlighting mutation of genes with established roles in MM, we identified a number of frequently acquired de novo coding mutations (e.g., FAM46C, TRAF2, NF1, and $X B P 1)$, de novo translocation (MAP3K14) and preexisting mutations (e.g., TET2). Longer telomeres at relapse could be associated with treatment as observed in chronic myeloid leukemia ${ }^{57}$. Therapy targeting telomerase/telomeres should be further explored in $M M$ as lengthened telomeres may provide a mechanism for treatment resistance ${ }^{58}$.

By performing high-depth WGS, we have been able to better refine the patterns of genomic evolution at relapse in MM compared to previous studies ${ }^{3,4}$. Notably, the "branching evolution" and "differential clonal response" models described by Bolli et al. ${ }^{3}$ often co-occurred as one single model (Pattern 2) in our analysis. Additionally, we did not find evidence for an association between $t(11 ; 14)$ MM with a "no change/linear" model ${ }^{3}$. The study by Jones et al. which included a small number of overlapping cases failed to identify Pattern 2 whereby a subclone survives treatment and expands at relapse ${ }^{4}$. Insights into tumour evolution has the potential to inform clinical decisions ${ }^{59}$. 


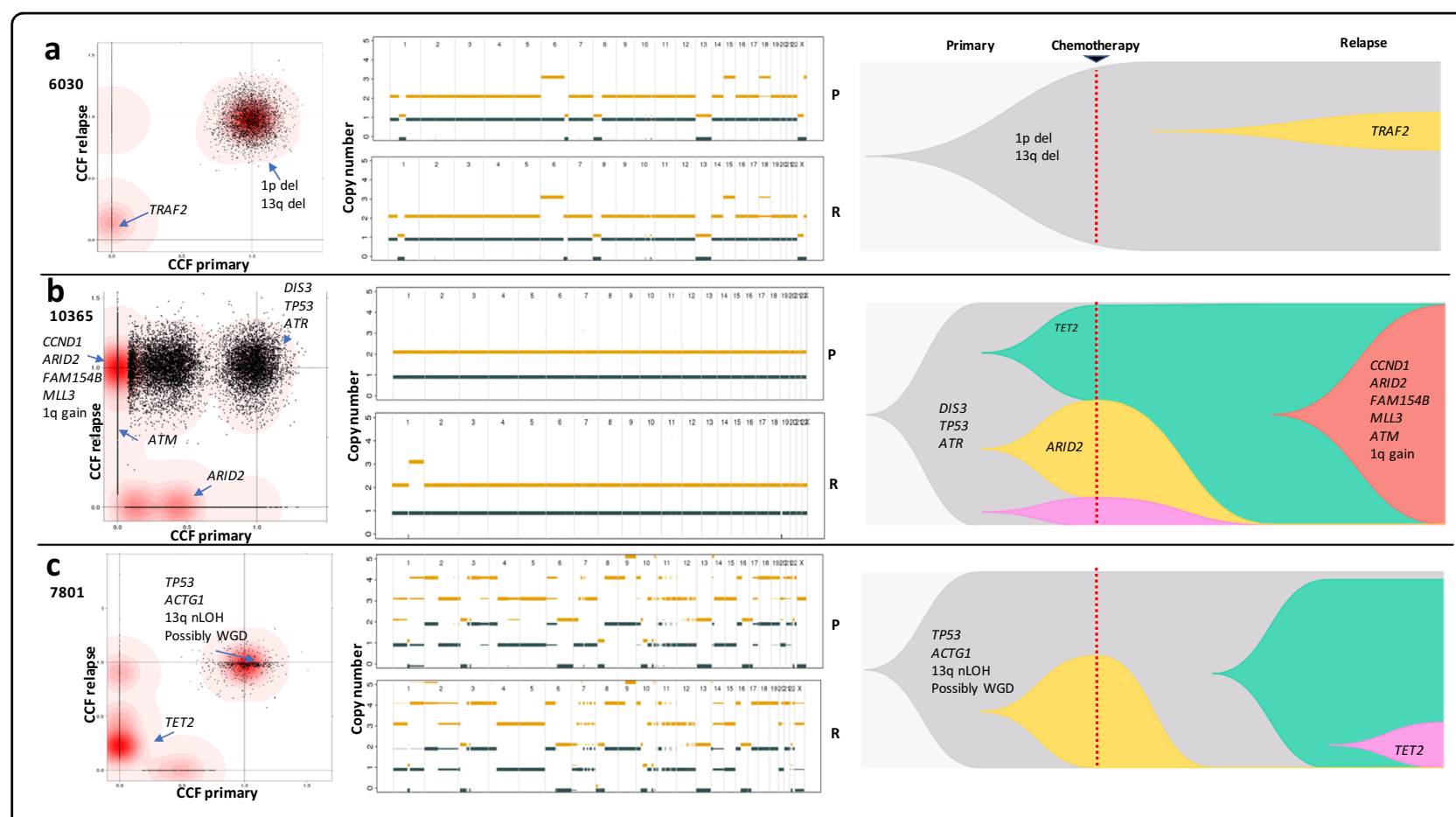

Fig. 3 Evolutionary trajectories of relapse. a Pattern 1 (3/24), dominant clone in primary survives treatment and gains additional mutations at relapse; b Pattern 2 (4/24), subclone in primary survives treatment and expands to become dominant clone at relapse; c Pattern 3 (17/24), eradication or decrease in frequency of one or more clones in primary and emergence of new clones not previously detected in primary. Left panels, twodimensional density plots showing clustering of mutations by cancer cell fraction (CCF) in primary and relapse tumours. Darker red areas indicate location of a high posterior probability of a cluster. Clusters are annotated with coding driver mutations and major copy number alterations. Pattern 1: no disappearance of primary clusters on the horizontal axis accompanied by appearance of new clusters on the vertical axis. Pattern 2: existence of cluster positioned on the vertical top and horizontal centre. Pattern 3: disappearance of clusters on the horizontal axis accompanied by appearance of clusters on the vertical axis. Central panels, chromosomal copy-number profiles of primary (upper) and relapse (lower) tumours. Thick and thin lines represent clonal and sub-clonal copy number states respectively. Yellow and dark blue lines denote total and minor copy number alleles. Right panels, Muller plots of evolutionary trajectories. P primary, R relapse. WGD Whole genome duplication.

"Evolutionary herding", in which clonal composition of tumours is tunnelled by a treatment to increase their sensitivity to another treatment, has been proposed as a strategy to combat treatment-resistance in tumours ${ }^{60}$. Despite a limited number of samples, we found little evidence that the evolutionary trajectory of MM is solely dictated by molecular karyotype or significantly influenced by current therapeutic strategies, questioning the viability of "evolutionary herding" in controlling drug resistance in MM. It was however noteworthy that Pattern 2 was associated with significant shorter time to relapse. Going forward, further strategies should be explored to accurately predict tumour dynamics and tailor patient therapy $^{61}$.

Higher proportion of $C \cdot G>G \cdot C$ at relapse is associated with DNA damage by oxidative stresses ${ }^{62}$, possibly due to oncogene activation and/or enhanced metabolism in relapsed $\mathrm{MM}^{63}$. AID/APOBEC activity contributes to increased mutational burden and associated with shorter time to relapse. APOBEC mutagenesis has been shown to promote survival and therapy escape in cancer through driving subclonal diversity, immune evasion, and genomic instability ${ }^{64}$. Collectively, these data suggest APOBEC family enzymes as potential therapeutic targets for treatment-resistance MM.

Inevitably, due to technical limitations, our ability to detect mutations in rare cells (mostly related to currently achievable levels of coverage with WGS) and spatial sampling constraints, our models potentially underestimate clonal heterogeneity in MM. We did however observe the loss of primary tumour clones at relapse in 21 of 24 cases, suggesting that some subclones are eradicated by therapy (Supplementary Fig. 22). Nevertheless, treatment failed to eradicate the founding clone in all cases. Our data also imply the acquisition of new mutations, which subsequently undergo selection and clonal expansion, potentially contributing to disease progression. It is likely that some mutations gained at relapse may alter the growth properties of MM cells, or confer resistance to additional chemotherapy.

Presently strategies to improve the poor cure rates of relapsing $M M$ are limited. The forces shaping the 
evolutionary trajectory of MM have relevance to informing patient management. Williams et al. proposed that following a "big bang", neutral evolution is a major feature of many cancers ${ }^{65}$. Application of same model to MM exome sequencing data suggested that neutral evolution is also a significant feature of $\mathrm{MM}^{66}$. Serious criticism has however been levelled at the assumptions on which the Williams et al. model is predicated ${ }^{67-70}$. In the light of such critique, as well as findings from our current WGS analysis and MM sequencing studies performed by other researchers $^{71}$, it is apposite to reappraise the role of neutral evolution in MM. It seems highly unlikely neutral evolution is a dominant evolutionary force in MM and its evolutionary trajectory is essentially Darwinian-shaped by selection and subsequent expansion of diverse clones in patients.

MM cells routinely acquire a small number of additional mutations at relapse, and some of these mutations may contribute to clonal selection and therapy resistance. While mutations in CRBN and associated genes have been implicated as a mechanism of acquired drug resistance to IMiDs, our analysis suggests mutation per se is unlikely to be a universal basis of acquired IMiD resistance. This does not preclude epigenetic alterations, which are a feature of relapse influencing drug transport, escape from apoptosis, and dysregulated intracellular signalling pathways, all of which can contribute to resistance ${ }^{72}$.

Here, we have demonstrated that relapsed MM harbour significantly more mutations than primary tumours and clonal selection of mutations occurs at relapse, which are accompanied by subclonal heterogeneity. Theoretically, these data provide a rationale for identifying diseasecausing mutations for MM, which may be amenable to targeted therapies to avoid the use of cytotoxic drugs, many of which are mutagens. However, it remains to be determined whether the current arsenal of therapies directed against downstream effectors of mutated genes will be effective given that the MM genome in an individual patient is likely to be continuously evolving. It is conceivable that in the near future, chemotherapy-based regimens may be relegated to fifth or sixth line treatment after patients have failed proteasome inhibitors, IMiDs and/or immunotherapy. Although speculative, however successful immunotherapy will be in an individual patient, Darwinian evolution of MM would imply that such therapy is unlikely to affect cure. It is therefore likely that eradication of the founding clone, as well as all of its subclones, will be required to effect complete cure.

\footnotetext{
Acknowledgements

This work was supported by grants from Myeloma UK, Bloodwise and Cancer Research UK (C1298/A8362). We are grateful to the NCRI Haemato-oncology subgroup and to all investigators for recruiting patients to Myeloma XI. These data were generated as part of the Myeloma XI trial. M.K. is supported by a fellowship from the David Forbes-Nixon Foundation.
} 


\section{Author details}

'Division of Genetics and Epidemiology, The Institute of Cancer Research, London SM2 5NG, UK. ²Division of Molecular Pathology, The Institute of Cancer Research, London SM2 5NG, UK. ${ }^{3}$ Department of Haematology, University of Newcastle, Newcastle Upon Tyne, UK. ${ }^{4}$ Perlmutter Cancer Center, NYU Langone Health, New York, USA. ${ }^{5}$ Leeds Institute of Cancer and Pathology, University of Leeds, Leeds, UK

\section{Author contributions}

P.H.H., M.K., and R.S.H. conceived and designed the study; P.H.H., A.J.C., D.C., and B.K. performed bioinformatics; A.S. and S.K. generated data; G.J., G.J.M., and G.C. provided samples; P.H.H. and R.S.H. wrote the manuscript with contributions from A.J.C., M.K., and D.C. All authors reviewed the final manuscript.

\section{Data availability}

Raw promoter capture $\mathrm{Hi}-\mathrm{C}$ data for naïve $\mathrm{B}$-cells were obtained from European Genome-Phenome Archive (EGA; accession code EGAS00001001911). Replication timing data for B-lymphocytes was downloaded from Replication Domain Database ${ }^{34}$. Raw WGS data generated as part of this study can be accessed through EGA accession code EGAD00001005491.

\section{Conflict of interest}

The authors declare that they have no conflict of interest.

\section{Publisher's note}

Springer Nature remains neutral with regard to jurisdictional claims in published maps and institutional affiliations.

Supplementary Information accompanies this paper at (https://doi.org/ 10.1038/s41408-020-00367-2).

Received: 30 June 2020 Revised: 15 September 2020 Accepted: 28 September 2020

Published online: 14 October 2020

\section{References}

1. Manier, S. et al. Genomic complexity of multiple myeloma and its clinical implications. Nat. Rev. Clin. Oncol. 14, 100-113 (2017).

2. Shah, $V$. et al. Prediction of outcome in newly diagnosed myeloma: a metaanalysis of the molecular profiles of 1905 trial patients. Leukemia 32, 102-110 (2018).

3. Bolli, N. et al. Heterogeneity of genomic evolution and mutational profiles in multiple myeloma. Nat. Commun. 5, 2997 (2014).

4. Jones, J. R. et al. Clonal evolution in myeloma: the impact of maintenance lenalidomide and depth of response on the genetics and sub-clonal structure of relapsed disease in uniformly treated newly diagnosed patients. Haematologica 104, 1440-1450 (2019).

5. Walker, B. A. et al. A compendium of myeloma-associated chromosomal copy number abnormalities and their prognostic value. Blood 116, e56-e65 (2010).

6. Kaiser, M. F. et al. A TC classification-based predictor for multiple myeloma using multiplexed real-time quantitative PCR. Leukemia 27, 1754-1757 (2013).

7. Hoang, P. H. et al. Whole-genome sequencing of multiple myeloma reveals oncogenic pathways are targeted somatically through multiple mechanisms. Leukemia 32, 2459-2470 (2018).

8. Langmead, B., Trapnell, C., Pop, M. \& Salzberg, S. L. Ultrafast and memoryefficient alignment of short DNA sequences to the human genome. Genome Biol. 10, R25 (2009).

9. Lee, S. et al. NGSCheckMate: software for validating sample identity in nextgeneration sequencing studies within and across data types. Nucleic Acids Res. 45, e103 (2017).

10. Costello, M. et al. Discovery and characterization of artifactual mutations in deep coverage targeted capture sequencing data due to oxidative DNA damage during sample preparation. Nucleic Acids Res. 41, e67 (2013).
11. Karczewski, K. J. et al. The mutational constraint spectrum quantified from variation in 141,456 humans. Nature 581, 434-443 (2020).

12. Letouze, E. et al. Mutational signatures reveal the dynamic interplay of risk factors and cellular processes during liver tumorigenesis. Nat. Commun. 8, 1315 (2017).

13. Nik-Zainal, S. et al. The life history of 21 breast cancers. Cell 149, 994-1007 (2012).

14. Soong, D. et al. CNV Radar: an improved method for somatic copy number alteration characterization in oncology. BMC Bioinform. 21, 98 (2020).

15. Favero, F. et al. Sequenza: allele-specific copy number and mutation profiles from tumor sequencing data. Ann. Oncol. 26, 64-70 (2015).

16. Shen, R. \& Seshan, V. E. FACETS: allele-specific copy number and clonal heterogeneity analysis tool for high-throughput DNA sequencing. Nucleic Acids Res. 44, e131 (2016).

17. Yuan, K. Macintyre, G., Liu, W. \& Markowetz, F. Ccube: a fast and robust method for estimating cancer cell fractions. bioRxiv, 484402, https://doi.org/ 10.1101/484402 (2018).

18. Wala, J. A. et al. Selective and mechanistic sources of recurrent rearrangements across the cancer genome. bioRxiv, 187609, https://doi.org/10.1101/187609 (2017).

19. Chen, X. et al. Manta: rapid detection of structural variants and indels for germline and cancer sequencing applications. Bioinformatics 32, 1220-1222 (2016).

20. Layer, R. M., Chiang, C., Quinlan, A. R. \& Hall, I. M. LUMPY: a probabilistic framework for structural variant discovery. Genome Biol 15, R84 (2014).

21. Rausch, T. et al. DELLY: structural variant discovery by integrated paired-end and split-read analysis. Bioinformatics 28, i333-i339 (2012).

22. Cortés-Ciriano, I. et al. Comprehensive analysis of chromothripsis in 2,658 human cancers using whole-genome sequencing. Nat. Genet. 52, 331-341 (2020).

23. Baca, S. C. et al. Punctuated evolution of prostate cancer genomes. Cell $\mathbf{1 5 3}$ 666-677 (2013).

24. Farmery, J. H. R., Smith, M. L., Diseases, N. B.-R. \& Lynch, A. G. Telomerecat: a ploidy-agnostic method for estimating telomere length from whole genome sequencing data. Sci. Rep. 8, 1300 (2018).

25. Hoang, P. H., Cornish, A. J., Dobbins, S. E., Kaiser, M. \& Houlston, R. S. Mutational processes contributing to the development of multiple myeloma. Blood Cancer J. 9, 60 (2019).

26. Martincorena, I. et al. Universal patterns of selection in cancer and somatic tissues. Cell 171, 1029-1041 (2017).

27. Walker, B. A. et al. Identification of novel mutational drivers reveals oncogene dependencies in multiple myeloma. Blood 132, 587-597 (2018).

28. Harrow, J. et al. GENCODE: the reference human genome annotation for The ENCODE Project. Genome Res. 22, 1760-1774 (2012).

29. Javierre, B. M. et al. Lineage-specific genome architecture links enhancers and non-coding disease variants to target gene promoters. Cell 167, 1369-1384 (2016).

30. Wingett, S. et al. HiCUP: pipeline for mapping and processing Hi-C data. F1000Res. 4, 1310 (2015)

31. Cairns, J. et al. CHiCAGO: robust detection of DNA looping interactions in capture Hi-C data. Genome Biol. 17, 127 (2016).

32. Melton, C., Reuter, J. A., Spacek, D. V. \& Snyder, M. Recurrent somatic mutations in regulatory regions of human cancer genomes. Nat. Genet. 47, 710-716 (2015).

33. Weddington, N. et al. ReplicationDomain: a visualization tool and comparative database for genome-wide replication timing data. BMC Bioinform. 9, 530 (2008).

34. Sima, J. et al. Identifying cis elements for spatiotemporal control of mammalian DNA replication. Cell 176, 816-830 (2019).

35. Rheinbay, E. et al. Recurrent and functional regulatory mutations in breast cancer. Nature 547, 55-60 (2017).

36. Kortum, K. M. et al. Targeted sequencing of refractory myeloma reveals a high incidence of mutations in CRBN and Ras pathway genes. Blood 128, 1226-1233 (2016).

37. Gooding, S. et al. Multiple cereblon genetic changes associate with acquired resistance to lenalidomide or pomalidomide in multiple myeloma. Blood https://doi.org/10.1182/blood.2020007081 (2020). Epub ahead of print.

38. Aktas Samur, A. et al. Deciphering the chronology of copy number alterations in multiple myeloma. Blood Cancer J. 9, 39 (2019).

39. Caravagna, G. et al. Subclonal reconstruction of tumors by using machine learning and population genetics. Nature Genetics 52, 898-907 (2020). 
40. Shinde, J. et al. Palimpsest: an R package for studying mutational and structural variant signatures along clonal evolution in cancer. Bioinformatics $\mathbf{3 4}$ 3380-3381 (2018).

41. Alexandrov, L. B. et al. Signatures of mutational processes in human cancer. Nature 500, 415-421 (2013).

42. Rosenthal, R., McGranahan, N., Herrero, J., Taylor, B. S. \& Swanton, C. DeconstructSigs: delineating mutational processes in single tumors distinguishes DNA repair deficiencies and patterns of carcinoma evolution. Genome Biol. 17, 31 (2016).

43. Maura, F. et al. A practical guide for mutational signature analysis in hematological malignancies. Nat. Commun. 10, 2969 (2019).

44. Maura, F. et al. Genomic landscape and chronological reconstruction of driver events in multiple myeloma. Nat. Commun. 10, 3835 (2019).

45. Kaufmann, H. et al. Both IGH translocations and chromosome $13 q$ deletions are early events in monoclonal gammopathy of undetermined significance and do not evolve during transition to multiple myeloma. Leukemia $\mathbf{1 8}$, 1879-1882 (2004)

46. Weinhold, N. et al. Clonal selection and double-hit events involving tumor suppressor genes underlie relapse in myeloma. Blood 128, 1735-1744 (2016)

47. Ding, L. et al. Clonal evolution in relapsed acute myeloid leukaemia revealed by whole-genome sequencing. Nature 481, 506-510 (2012).

48. Walker, B. A. et al. Intraclonal heterogeneity and distinct molecular mechanisms characterize the development of $\mathrm{t}(4 ; 14)$ and $\mathrm{t}(11 ; 14)$ myeloma. Blood 120, 1077-1086 (2012).

49. Corre, J. et al. Multiple myeloma clonal evolution in homogeneously treated patients. Leukemia 32, 2636-2647 (2018).

50. Egan, J. B. et al. Whole-genome sequencing of multiple myeloma from diagnosis to plasma cell leukemia reveals genomic initiating events, evolution, and clonal tides. Blood 120, 1060-1066 (2012).

51. Keats, J. J. et al. Clonal competition with alternating dominance in multiple myeloma. Blood 120, 1067-1076 (2012).

52. Chapman, M. A. et al. Initial genome sequencing and analysis of multiple myeloma. Nature 471, 467-472 (2011).

53. Alvarez-Prado, A. F. et al. A broad atlas of somatic hypermutation allows prediction of activation-induced deaminase targets. J. Exp. Med. 215, 761-771 (2018).

54. Hochhaus, A. et al. Molecular and chromosomal mechanisms of resistance to imatinib (STI571) therapy. Leukemia 16, 2190-2196 (2002).

55. Lohr, J. G. et al. Widespread genetic heterogeneity in multiple myeloma: implications for targeted therapy. Cancer Cell 25, 91-101 (2014).
56. Maura, F. et al. Role of AID in the temporal pattern of acquisition of driver mutations in multiple myeloma. Leukemia 13, 1-5 (2019).

57. Brümmendorf, T. H. et al. Normalization of previously shortened telomere length under treatment with imatinib argues against a preexisting telomere length deficit in normal hematopoietic stem cells from patients with chronic myeloid leukemia. Ann. N. Y. Acad. Sci. 996 26-38 (2003).

58. Lipinska, N. et al. Telomerase and drug resistance in cancer. Cell Mol. Life Sci. 74, 4121-4132 (2017).

59. Fittall, M. W. \& Van Loo, P. Translating insights into tumor evolution to clinical practice: promises and challenges. Genome Med. 11, 20 (2019).

60. Acar, A. et al. Exploiting evolutionary herding to control drug resistance in cancer. bioRxiv, 566950, https://doi.org/10.1101/566950 (2019).

61. Lipinski, K. A. et al. Cancer evolution and the limits of predictability in precision cancer medicine. Trends Cancer 2, 49-63 (2016).

62. Kino, K. \& Sugiyama, H. UVR-induced G-C to C-G transversions from oxidative DNA damage. Mutat. Res. 571, 33-42 (2005).

63. Liou, G. Y. \& Storz, P. Reactive oxygen species in cancer. Free Radic. Res. 44, 479-496 (2010)

64. Venkatesan, S. et al. Perspective: APOBEC mutagenesis in drug resistance and immune escape in HIV and cancer evolution. Ann. Oncol. 29, 563-572 (2018).

65. Williams, M. J., Werner, B., Barnes, C. P., Graham, T. A. \& Sottoriva, A. Identification of neutral tumor evolution across cancer types. Nat. Genet. 48, 238-244 (2016).

66. Johnson, D. C. et al. Neutral tumor evolution in myeloma is associated with poor prognosis. Blood 130, 1639-1643 (2017).

67. Tarabichi, M. et al. Neutral tumor evolution? Nat. Genet. 50, 1630-1633 (2018).

68. McDonald, T. O., Chakrabarti, S. \& Michor, F. Currently available bulk sequencing data do not necessarily support a model of neutral tumor evolution. Nat Genet. 50, 1620-1623 (2018).

69. Balaparya, A. \& De, S. Revisiting signatures of neutral tumor evolution in the light of complexity of cancer genomic data. Nat. Genet. 50, 1626-1628 (2018).

70. Wang, H.-Y. et al. Is the evolution in tumors Darwinian or non-Darwinian? Natl Sci. Rev. 5, 15-17 (2017).

71. Bahlis, N. J. Darwinian evolution and tiding clones in multiple myeloma. Blood 120, 927-928 (2012).

72. Pinto, $\mathrm{V}$. et al. Multiple myeloma: available therapies and causes of drug resistance. Cancers $\mathbf{1 2}, 407$ (2020). 